measures were taken for a 4249 men aged 70 years and above between 2001 and 2004. We examined the association between 1-year average pollutant concentrations (fine particulate matter, black carbon, nitrogen dioxide, and nitrogen oxides) with systolic blood pressure, diastolic blood pressure, cholesterol (high and low-density lipoprotein), triglycerides, high sensitivity C-reactive protein, and total homocysteine. Linear regression analyses were performed, with adjustment for potential confounders, as well as assessment of effect modification.

Results

After controlling for potential confounders, we found that a $2.25 \mu \mathrm{g} /$ $\mathrm{m} 3$ higher exposure to fine particulate matter (PM2.5) was significantly associated with a 1.4 percent lower high-density cholesterol $(95 \%$ confidence interval: -2.5 to -0.2 ) and 4.2 percent higher serum triglycerides (95\% confidence interval: 1.7 to 6.7). The strength of these associations was not modified by age, BMI, or socioeconomic status. We found no evidence of an association between any of the remaining risk factors with measures of outdoor air pollution.

Conclusions

These findings indicate that long-term exposure to fine particulate matter is associated with elevated serum triglycerides and decreased HDL cholesterol. This warrants further investigation to better understand the potential role that these risk factors have in mediating effects of air pollution on CVD.

\section{Indoor Exposure to Selected Air Pollutants and Associated Health Effects: A Global Review}

Vardoulakis $S^{1}$, Davis $A^{1}$, Steinle $S^{1}$, Sleeuwenhoek $A^{1}$, Galea $K^{1}$, Dixon $K^{1}$, Crawford $J^{1}$

${ }^{1}$ Institute of Occupational Medicine

TPS 901: Indoor air pollution, Exhibition Hall, Ground floor, August 28, 2019, 3:00 PM - 4:30 PM

Background: There is increasing awareness that the quality of the indoor environment affects our health and wellbeing. Indoor air quality in particular has an impact on multiple health outcomes, including respiratory and cardiovascular illness, allergic symptoms, cancers, and premature mortality.

Methods: We carried out a global systematic literature review on indoor air quality, related household characteristics, occupancy patterns, and health effects. Indoor exposure levels and determinants, indoor/ outdoor ratios, and emission sources were extracted from 144 studies (from 31 countries). The most studied pollutants were particulate matter (PM2.5), nitrogen dioxide (NO2), Volatile Organic Compounds (VOC), and Polycyclic Aromatic Hydrocarbons (PAH).

Results: Indoor PM2.5 sources include smoking, cooking, heating, use of incense/candles, cleaning, housework, and movement of people. Outdoor air is a major PM2.5 source in rooms with natural ventilation in roadside households. Eliminating indoor smoking and switching from coal to gas or electricity for cooking substantially reduces indoor PM2.5. Major sources of NO2 indoors are unvented gas heaters and cookers. Predictors of indoor NO2 are ventilation, season, and outdoor NO2 levels. VOC are emitted from a wide range of indoor and outdoor sources, including smoking, solvent use, renovations, and household products. Formaldehyde levels are higher in newer houses and in the presence of new furniture, while PAH levels are higher in smoking households.

Conclusions: Household characteristics and occupant activities play a large role in indoor exposure, particularly cigarette smoking for PM2.5, gas appliances for NO2, and household products for VOC. Home location near high traffic density roads, redecoration, and small house size, contribute to high indoor air pollution. Formaldehyde is typically higher indoors than outdoors, with indoor levels correlated positively with temperature and negatively with age of building. In most studies, air exchange rates are negatively associated with indoor air pollution. These findings can improve residential indoor air quality interventions.

\section{Insights into the epidemiology of heat-related work injuries: a mixed-methods analysis of workers' compensation claims and stakeholder perspectives}

Varghese $B^{1}$, Pisaniello $D^{1}$, Hansen $A^{1}$, Barnett $A^{2}$, Williams $S^{1}$, Bi $P^{1}$, Hanson-Easey $S^{1}$, Heyworth $J^{3}$, Sim $M^{4}$, Nitschke $M^{5}$, Rowett $S^{6}$

${ }^{1}$ The University of Adelaide, ${ }^{2}$ Queensland University of Technology, ${ }^{3}$ The University of Western Australia, ${ }^{4}$ Monash University, ${ }^{5}$ SA Health, ${ }^{6}$ SafeWork $S A$

TPS 791: Occupational health 1, Exhibition Hall, Ground floor, August 26, 2019, 3:00 PM - 4:30 PM

Background/Aim:

Hot working conditions can lead to serious illness and increased risk of work-related injuries. The aim of this study was to better understand the heat-injury phenomenon in workplaces to inform prevention measures.

Methods:

A mixed-methods research approach was adopted to explore the nature and determinants of heat-related occupational injuries. The quantitative phase consisted of investigating the impact of heat exposure (maximum temperature and heatwave) on workers' compensation (WC) claims in four Australian cities with temperate (Adelaide, Melbourne and Perth) and sub-tropical (Brisbane) climates. National surveys of health and safety professionals and representatives were undertaken to gauge their perceptions on determinants of heat-related work injuries. The qualitative component consisted of analyzing data collected from sources including interviews, surveys, and a telephone complaints database.

Results:

Analysis of WC data showed injuries increased during moderate and extremely hot temperatures in Adelaide and Melbourne, while a reduction in risk was seen at the coolest temperatures in Brisbane and Perth. During moderate/high-severity heatwave days injuries increased compared to non-heatwave days in each city with the highest effect in Brisbane. Contributing factors for heat-related injuries were reported to be: fatigue and dehydration, issues with personal protective equipment (PPE), lack of heat awareness training, poor management practices, concerns over productivity loss and inflexibility of supervisors. Recommendations for prevention include more awareness and training, better planning of work and rest breaks, better designed PPE, rotation of workers or tasks, and if possible, having cease-work temperatures.

Conclusions:

This study yielded insights into the nature and distribution of occupational injuries that occur in hot conditions. The evidence suggests that the underlying mechanism for physical injury is an interaction of existing physical hazards and increased worker susceptibility in hot conditions. Prevention efforts need to consider these multi-factorial determinants particularly in light of warmer conditions predicted due to climate change.

\section{The associations of geomagnetic storms, fast solar wind, and stream interaction regions with cardiovascular characteristic in patients with acute coronary syndrome} Kiznys $D^{1}$, Vencloviene $\mathrm{J}^{1,2}$, Milvidaite $I^{2}$

${ }^{1}$ Vytautas Magnus University, ${ }^{2}$ Lithuanian University of Health Sciences

TPS 771: Diet and lifestyle, Exhibition Hall, Ground floor, August 26, 2019, 3:00 PM - 4:30 PM

Background/Aim: Solar activity and other space weather events affect the Earth's atmosphere by causing geomagnetic activity and change in weather pattern. A lot of publications have confirmed that 